\title{
Intérêts et techniques de greffe autogène pour la reconstruction de défauts osseux verticaux postérieurs mandibulaires
}

\author{
J. Raidot (Strasbourg), F. Scheid (Strasbourg), P.O. Sage (Strasbourg), J.M. Offerle \\ (Strasbourg), P. Keller (Strasbourg)
}

La thérapeutique implantaire se présente aujourd'hui comme une solution de choix pour une réhabilitation prothétique esthétique et fonctionnelle. Cependant un volume osseux minimum est nécessaire. Dans le cas d'édentement mandibulaire postérieur, certains défauts osseux ne permettent pas la mise en place d'implants sans passer par une reconstruction du volume. Les greffes osseuses verticales dans le secteur posté rieur mandibulaire sont décrites comme une procé dure complexe. Différentes techniques aboutissant à l'augmentation du volume osseux sont décrites. Elles sont toutes constituées d'un coffrage, réalisé à partir de bloc autogène, d'une membrane résorbable ou non, avec ou sans armature en titane. Dans ce coffrage est disposé un comblement composé soit d'os particulé autogène, xénogène, ou d'un mélange d'os avec des biomatériaux. Les complications décrites sont variables en fonction de la technique utilisée. Le but de ce travail est de faire le point sur les différents avantages et inconvénients de ces techniques. Pour les comparer nous ferons une revue de la littérature. Nous mettrons ensuite en avant l'intérêt de l'utilisation de l'os autogène seul par l'étude d'une série de différents cas cliniques.

1 - Fouad Khoury, Hadi Antoun, Patrick Missika : Bone augmentation in oral implantology Quintessence books. - Daniel Buser : 20 years of guided bone regeneration in implant dentistry Quintessence books. 